Method. We identified 50 out of 359 patients within our service who were admitted to psychiatric hospital over a one year period (between 01/11/2019-01/11/2020).

We looked at medication compliance, use of the Mental Health Act and accommodation status to compare between those with and without known dual diagnosis. We used frequency and length of admission as indicators of how successfully patients were being managed in the community and the cost to the hospital trust. Urine drug screening and referral to substance misuse services were chosen as markers of whether patients were being appropriately managed on admission.

Result. A higher percentage of patients with dual diagnosis were detained under the Mental Health Act compared to those without substance misuse ( $89 \%$ versus $72 \%$ ). They were more likely to have no fixed abode (28\% versus $13 \%$ ) and be non-compliant with treatment pre-admission ( $83 \%$ versus $56 \%$ ). Patients with dual diagnosis also had a higher number of hospital admissions, with a greater proportion having 3 admissions that year (11\% versus 3\%).

Only $50 \%$ of patients with known dual diagnosis had a urine drug screen performed on admission and just $25 \%$ of patients who were currently misusing substances were referred to specialist services by the inpatient team.

Conclusion. Our audit found that there are overall poorer outcomes for patients with dual diagnosis versus a psychiatric illness only. It is evident that integration of services will improve the care we are able to provide and reduce costs associated with multiple admissions to hospital.

We identified three key areas for improvement. Firstly, we advised on the need to improve documentation. Additionally, we recommend ensuring assessment of current drug misuse is done on admission, including performing simple tests such as urine drug screening. Finally, we highlighted the need to improve discussions about substance misuse with patients, within teams and between services, aiming for integrated and holistic care.

\section{Audit of ADHD medication prescription and monitoring in intellectual disability services, Greater Glasgow \& Clyde}

Neha Bansal* and Muzammil Hayat

NHS Greater Glasgow and Clyde

${ }^{*}$ Corresponding author.

doi: 10.1192/bjo.2021.218

Aims. Studies have shown that people with intellectual disability (ID) show a greater severity of attention deficit hyperactivity disorder (ADHD) symptoms and atypical presentation, as well as having a greater risk of developing comorbidities, such as challenging behaviour, anxiety, tic disorders and sleep problems. It is estimated that $1.5 \%$ of patients with ID will have a clinical diagnosis of ADHD.

The aim of this audit was to find whether individuals with ID and ADHD, who are prescribed medication for ADHD are adequately monitored and reviewed in accordance with the ADHD medication prescription guidance by NICE and the Royal College of Psychiatrists (RCPsych).

Method. This audit looked at ADHD medication prescription for the ID population within Greater Glasgow \& Clyde NHS. This is the 6th audit cycle where electronic records (EMIS) were analysed between $28 / 9 / 19$ to $09 / 10 / 20$. (The 5 th cycle data collection period ended on 28/9/19). We collected data on all patients aged over 18 years.

An audit tool was developed to find whether the following were documented; patient demographics, physical health monitoring, symptom severity, medication dosage, side effects, need for ongoing treatment and frequency of review. 100\% of patients should have all components on the ADHD audit tool documented, as per NICE/ RCPsych prescription guidance.

Result. 32 patients were identified as being diagnosed with ADHD prescribed medication. One patient was impacted by the COVID-19 pandemic which meant that the required monitoring was not fully carried out. The age ranged from 18 to 56 years. $75 \%$ had mild intellectual disability, $19 \%$ had moderate and $6 \%$ had severe, with no cases of profound intellectual disability. Blood Pressure/pulse was recorded in $84 \%$ of patients. Height/weight/ BMI was recorded in $81 \%$ of patients. $97 \%$ of patients had ADHD symptom severity, medication dosage, side effects, need for ongoing treatment and frequency of review recorded.

Conclusion. There is further scope for improvement in the monitoring and documentation of physical health observations, however there was a significant improvement compared to the previous cycle of the audit. Other aspects of monitoring and documentation appear to be recorded in almost $100 \%$ of patients. This finding emphasises the challenges of physical health monitoring and compliance in psychiatry as a whole. We need to continue to encourage awareness and education around the physical health risks to our patients, not only due to their comorbidities but also as a result of the psychotropic medications we prescribe them.

\section{Are patients self-referring to Lewisham Community}

Wellbeing (LCW) when advised to do so by the assessment and liaison psychiatry team?

Gautam Bhatia $^{1 \star}$, Thileepan Thevarajan ${ }^{1}$, Jadesh Manivannan ${ }^{2}$ and Danny Majidian ${ }^{2}$

${ }^{1}$ South London and Maudsley NHS Foundation Trust and ${ }^{2}$ medical student, Kings College London

${ }^{*}$ Corresponding author.

doi: 10.1192/bjo.2021.219

Aims. It is well-recognised by the RCPsych that mental illness is both a cause and consequence of social exclusion, and thus social inclusion is an important part of recovery and leads to better outcomes for patients.

The Lewisham Assessment and Liaison team Neighbourhood 4 (A\&L N4) is a CMHT service that acts as an intake team for all referrals into secondary care mental health services, with the purpose of assessment and brief intervention. Currently, if a patient is assessed to potentially benefit from our local social inclusion service, Lewisham Community Wellbeing (LCW), they are advised to self-refer. However, there is no follow-up as to whether patients go on to do this.

Therefore, this audit aimed to calculate:

How many patients are advised to self-refer to LCW (advised referral)

How many of these patients make the self-referral to LCW (completed referral)

Method. The electronic notes for patients who were accepted by the A\&L N4 team from July to September 2020 were retrospectively analysed to see if an LCW self-referral was advised. A list of these patients was then given to LCW to check whether they had self-referred.

Result. A\&L N4 worked with 82 patients during the study period. 16 patients were advised to self-refer to LCW- an advised referral rate of $19.5 \%$. There was notable month-to-month variation in the advised referral rate- $29.6 \%$ in July vs. $9.4 \%$ in September. 